\title{
Exebacase: A Novel Approach to the Treatment of Staphylococcal Infections
}

\author{
Matthew W. McCarthy ${ }^{1}$
}

Accepted: 3 February 2022 / Published online: 17 February 2022

(c) The Author(s) 2022

\begin{abstract}
Lysins are bacteriophage-derived enzymes that degrade essential components of bacteria. Exebacase (Lysin CF-301) is an attractive antimicrobial agent because it demonstrates rapid bacteriolytic activity against staphylococcal species, including Staphylococcus aureus, has a low resistance profile, eradicates biofilms, and acts synergistically with other antibiotics. Combinations including exebacase and standard of care antibiotics represent an alternative to antibiotic monotherapies currently used to treat invasive staphylococcal infections. This manuscript reviews what is known about exebacase and explores how this novel agent may be used in the future to treat human bacterial pathogens.
\end{abstract}

\section{Key Summary Points}

Lysins have emerged as antimicrobial agents owing to their potency and specificity for bacterial pathogens in comparison with antibiotics.

Exebacase is a first-in-class anti-staphyloccocal lysin that has applications for the treatment of a variety of clinical syndromes.

Exebacase is rapidly bacteriostatic, eradicates biofilms, and has demonstrated clinical efficacy in combination with other antibiotics against a variety of staphylococcal pathogens that produce syndromes ranging from bacteremia to osteomyelitis.

\section{Introduction}

Lysins are enzymes produced by bacteriophages to cleave the bacterial host's cell wall during the final stage of the lytic cycle, releasing newly replicated virus from the bacteria

Matthew W. McCarthy

mwm9004@med.cornell.edu

1 Weill Cornell Medical College, 525 East 68th Street, Box 130, New York, NY 10065, USA
[1-3]. Lysins have emerged as antimicrobial agents owing to their potency and specificity for bacterial pathogens in comparison with antibiotics, which remain perpetually susceptible to bacterial resistance and may be ineffective in the presence of biofilms [4, 5]. Many lysins are species- or subspecies-specific, which means that they have a narrow spectrum of antimicrobial activity, and are only effective against bacteria from which they were produced [6]. Narrow targeting of bacterial pathogens represents a departure from many of the current approaches to treating infectious diseases, which often rely on broad-spectrum antibiotics that may be associated with off-target effects, which may be harmful to the host.

CF-301 (henceforth known as exebacase) has been identified as a promising lysin for research and development due to its specificity for Staphylcoccous aureus, which is associated with substantial morbidity and mortality for both humans and animals [7]. Exebacase is the lead compound in this new class of antimicrobial agents and may ultimately serve to complement antibiotics in a variety of human syndromes $[8$, 9]. Exebacase's relevance for veterinary medicine is outside of the scope of this manuscript and will not be discussed further.

\section{Clinical Relevance}

Exebacase, a first-in-class anti-staphyloccocal lysin, was found to be bacteriolytic against $250 \mathrm{~S}$. aureus strains tested, including 120 methicillin-resistant S. aureus (MRSA) 
isolates [10]. In time-kill studies, exebacase reduced $S$. aureus 1000-fold within half an hour, compared with 6-12 h required by antibiotics, suggesting a potential role in the treatment of human bloodstream infections.

Exebacase is an attractive agent for the treatment of $S$. aureus bacteremia because it acts synergistically with two key human blood factors, human serum lysozyme (HuLYZ) and human serum albumin (HSA), which normally have no nascent anti-staphylococcal activity [10]. Combinations of exebacase with two commonly used anti-staphylococcal antibiotics, vancomycin or daptomycin, yielded synergy in vitro and improved survival in staphylococcal-induced bacteremia in a murine model, suggesting combinations including exebacase and standard-of-care antibiotics could serve as an alternative strategy to treat $S$. aureus bacteremia, which continues to be associated with substantial morbidity and mortality in both immunocompetent and immunocompromised patients [8].

Recent work has established proof-of-principal. In a phase II, superiority design study, investigators randomly assigned 121 human volunteers with $S$. aureus bacteremia/ endocarditis to receive a single dose of exebacase or placebo in addition to standard-of-care antibiotics [11]. The primary efficacy endpoint was clinical outcome (responder rate) on day 14 . Response rates on day 14 were $70.4 \%$ and $60.0 \%$ in the exebacase + antibiotics and antibiotics-alone groups, respectively (difference $=10.4,90 \%$ confidence interval $[\mathrm{CI}]-6.3$ to $27.2 ; p=0.31$ ). Rates of adverse events were similar in both groups. Thirty-day all-cause mortality rates were $9.7 \%$ and $12.8 \%$ in the exebacase plus antibiotics and antibiotics-alone groups, respectively.

The most intriguing results were found in the subgroup analysis. For MRSA-infected patients, treatment with exebacase was associated with a marked reduction $(21 \%)$ in the 30-day all-cause mortality, a 4-day reduction in median hospital length of stay, and a reduction in 30-day hospital readmission rates in MRSA-infected patients. Taken together, these findings suggest a potential role for exebacase in the treatment of bloodstream infections attributable to staphylococci, including MRSA; however, further studies are warranted to determine the optimal dose and duration of treatment.

Staphylococcus is among the most common causes of endovascular infections, including infective endocarditis (IE), which may occur in the setting of a bloodstream infection [12-14]. In an experimental aortic valve MRSA IE rabbit model, transthoracic echocardiography was utilized to evaluate the in vivo effect of exebacase on vegetation progression when combined with daptomycin (versus daptomycin alone) [8]. One dose of exebacase in addition to daptomycin cleared significantly more vegetation than daptomycin alone, and MRSA counts in the combination group were significantly lower than those of untreated controls $(p<0.0001)$ and the daptomycin-alone group $(p<0.0001)$. Findings from this animal model suggest that exebacase has potential applications to address staphylococcal bloodstream infections and infectious endocarditis, and further suggest that the agent may be useful for other types of infection.

\subsection{Osteomyelitis}

Bone infections with drug-resistant organisms also pose a therapeutic challenge $[15,16]$. Patients are often subjected to prolonged courses of intravenous antimicrobial therapy (6-8 weeks) and treatment failure is not uncommon [17]. In this setting, exebacase has emerged as a promising addition to the standard of care for difficult-to-treat infections. In an acute MRSA osteomyelitis model, rats receiving no treatment or treatment with daptomycin, exebacase, or daptomycin plus exebacase had means of 5.13, 4.09, 4.65, and 3.57 $\log 10$ colony forming units (CFU)/g of bone, respectively [9]. All rats receiving treatment had a smaller bacterial burden than untreated animals $(p \leq 0.0001)$, with daptomycin plus exebacase being more active than daptomycin alone $(p=0.0042)$ or exebacase alone $(p<0.001)$.

Exebacase also has the potential to be used in patients with prosthetic joint infections due to staphylococci. For example, in patients with relapsing prosthetic knee infection, the only surgical option is exchange of the prosthesis $[18,19]$; however, surgical exchange can be associated with loss of function and mortality [20-22]. In one small study, exebacase was used during arthroscopic knee debridement and implantation followed by suppressive tedizolid as salvage therapy in patients with prior prosthetic knee revisions complicated by relapsing knee infection [23]. Exebacase $(75 \mathrm{mg} / \mathrm{mL} ; 30 \mathrm{~mL}$ ) was administered directly into the joint during arthroscopy. No adverse events occurred in the four patients who underwent the procedure; all patients received daptomycin $8 \mathrm{mg} / \mathrm{kg}$ and linezolid $600 \mathrm{mg}$ twice daily (4-6 weeks) as primary therapy, followed by tedizolid $200 \mathrm{mg} /$ day as suppressive therapy. After more than 1 year of followup, the clinical outcome was favorable in two patients with resolution of septic arthritis.

\subsection{Biofilms}

Biofilms are comprised of surface-associated microbial cells within an extracellular matrix [24]. This microenvironment represents a protected mode of growth that allows cells to survive in hostile environments and to withstand a variety of threats, including antimicrobial agents, and treatment failure is common $[25,26]$. Biofilms form in a variety of human tissues, including bone, skin, cardiac tissue, and the upper respiratory, intestinal and urinary tracts [27]. Medical devices, such as intravenous catheters, prosthetic joints, and pacemakers, are frequent sites of biofilm formation and pose 
a therapeutic challenge [28, 29]. Addressing biofilm-related infections is an unmet medical need [25, 30, 31].

In order to evaluate exebacase in this microenvironment, minimum biofilm-eradicating concentration (MBEC) assays were performed on S. aureus strains [32]. The effectiveness of exebacase was demonstrated against $S$. aureus biofilms formed on catheters, glass polystyrene, and surgical mesh. In catheters, exebacase removed all biofilm within $60 \mathrm{~min}$ and killed all released bacteria by $6 \mathrm{~h}$. Mixed-species biofilms, formed by S. aureus and coagulase-negative Staphylococcus on several surfaces, were removed by exebacase and activity was greatly improved in combinations with the other agents, such as cell wall hydrolase lysostaphin.

A series of in vitro pharmacodynamic (PD) parameters, including the post-antibiotic effect (PAE), post-antibiotic sub-MIC effect (PA-SME), and sub-MIC effect (SME), were evaluated to determine how exebacase exposures impact the growth of staphylococci [33]. Mean PAE, PA-SME, and SME values up to $4.8,9.3$, and $9.8 \mathrm{~h}$, respectively, were observed against 14 staphylococcal strains tested in human serum; a mouse thigh infection model demonstrated in vivo growth delays of more than $19 \mathrm{~h}$, suggesting that reductions in bacterial fitness and virulence may substantially enhance exebacase efficacy.

Taken together, these studies indicate that exebacase is effective at treating a variety of staphylococci in vitro and in vivo [7, 34-36]. Activity against other pathogens, such as Streptococcus pyogenes, $S$. agalactiae, and $S$. dysgalactiae remains strong but activity against other species is highly variable [34, 37-39]. Although human studies are limited, emerging data suggest that there may be a therapeutic role for exebacase in combination with existing antimicrobial therapy, with a clinical niche to address difficult-to-eradicate infections such as catheter-associated infections, endocarditis, prosthetic joint infections, and bacteremia due to staphylococci $[8,9,40]$. However, further studies are warranted to more fully categorize the safety and efficacy of this new agent in the treatment of biofilms, bone and joint infections, and endocarditis. For now, exebacase appears most promising for the treatment of staphylococcal bloodstream infections.

\section{Future Directions}

The challenge of antimicrobial drug resistance began soon after the discovery of the first antibiotics. Bacteria are continuously evolving to evade and withstand the commercially available antibiotics and novel treatment options are urgently needed. Bacteriophage-derived lysins are cellwall hydrolytic enzymes that represent a new approach to address the expanding threat of antimicrobial resistance [6, $41,42]$. Exebacase is a recombinantly produced lysin that is rapidly bacteriocidal, eradicates biofilms, and has demonstrated clinical efficacy in combination with other antibiotics against a variety of staphylococcal pathogens that produce syndromes ranging from bacteremia to osteomyelitis.

In vitro synergy for anti-biofilm activity of exebacase has been demonstrated with vancomycin, rifampin, and daptomycin against coagulase-negative Staphylocci (S. epidermidis) strains responsible for bone and joint infections of the knee, hip and shoulder. These data add to the existing evidence supporting the potential for exebacase to treat infections of prosthetic joints, although further studies are warranted. In the more immediate future, exebacase may serve as a novel treatment option for patients with bloodstream infections due to staphylococci. Recent work highlights the potential.

In October 2021, a phase II study was presented at IDWeek demonstrating that in patients with $S$. aureus bacteremia, exebacase used in addition to standard-of-care antibiotics resolved clinical symptoms more quickly than standard-of-care alone ( 3 days vs. 6 days) [oral presentation]. This randomized, double-blind, placebo-controlled study of 86 patients randomly assigned volunteers in a 2:1 ratio to a 2-h infusion of exebacase or placebo in addition to standard-ofcare antibiotics. Among patients with MRSA bacteremia, the median time to symptom resolution was 3 days for those who received exebacase, compared with 7 days in patients who received standard-of-care alone. Patients with MRSA bacteremia showed greater symptom resolution $(94.1 \%)$ than those treated with standard of care alone (81.8\%).

Exebacase is a direct lytic agent now in phase III of clinical development. In addition to the attributes described above, exebacase has a minimal tendency for the development of resistance, no cross-resistance with antibiotics, and an extended in vitro and in vivo PAE. The agent is also active in vitro in pulmonary surfactant, suggesting a potential role in the treatment of bacterial pneumonia and other respiratory diseases for which treatment options remain scarce.

For example, severe disease during influenza infection may be the result of secondary bacterial pneumonia due to S. aureus $[43,44]$. Viruses such as influenza A can disrupt physiological barriers and alter immunologic responses in humans, thereby altering the function of multi-protein inflammasomes, leading to increased susceptibility to staphylococci $[45,46]$. The ability of staphylococcal pathogens to thrive under physiologic conditions is also associated with their capacity to form biofilms in the respiratory mucous membranes, rendering many antibiotics ineffective [47, 48]. Co-infection has been a major cause of mortality during influenza pandemics and better treatment options are urgently needed [49].

Nasal carriage of $S$. aureus is a risk factor for secondary staphylococcal pneumonia in influenza A virus-infected hosts. In a mouse model of lethal S. aureus lung infection, 
exebacase was found to be efficacious alone and synergistic with daptomycin, potentially restoring the antimicrobial properties of an antibiotic that is known to become inactivated in the presence of pulmonary surfactant [37]. This approach may have particular relevance for patients with influenza pneumonia who subsequently develop staphylococcal pneumonia, which is associated with both acute and chronic lung injury as well as substantial mortality.

In the years ahead, exebacase may become an important addition to the therapeutic arsenal of agents used to treat invasive infections due to staphylocci, including bloodstream infections, endocarditis, lung infections, catheter-associated infections, and bone and prosthetic joint infections [9, 33, $36,50]$. Optimizing its use in clinical practice will be the focus of both basic and clinical research for years to come.

\section{Declarations}

Funding No funding was received to assist in the preparation of this manuscript.

Conflicts of Interest Matthew W. McCarthy has no declarations to declare and reports no conflicts of interest. This work was conducted in compliance with international ethical standards.

Consent to participate Not applicable.

Consent for publication Not applicable.

Availability of data and material Not applicable.

Code availability Not applicable.

Author contributions Matthew W. McCarthy was responsible for all aspects of this manuscript.

Open Access This article is licensed under a Creative Commons Attribution-NonCommercial 4.0 International License, which permits any non-commercial use, sharing, adaptation, distribution and reproduction in any medium or format, as long as you give appropriate credit to the original author(s) and the source, provide a link to the Creative Commons licence, and indicate if changes were made. The images or other third party material in this article are included in the article's Creative Commons licence, unless indicated otherwise in a credit line to the material. If material is not included in the article's Creative Commons licence and your intended use is not permitted by statutory regulation or exceeds the permitted use, you will need to obtain permission directly from the copyright holder. To view a copy of this licence, visit http://creativecommons.org/licenses/by-nc/4.0/.

\section{References}

1. Fischetti VA, Gotschlich EC, Bernheimer AW. Purification and physical properties of group $C$ streptococcal phage-associated lysin. J Exp Med. 1971;133(5):1105-17.
2. Schuch R, Nelson D, Fischetti VA. A bacteriolytic agent that detects and kills Bacillus anthracis. Nature. 2002;418(6900):884-9.

3. Winkler-Helmrich C. Lysin sensitivity and antibiotic resistance of types of Shigella [in German]. Zentralbl Bakteriol Orig. 1955;163(2-3):118-31.

4. Hallander HO. Characterization and partial purification of staphylococcal delta-lysin. Acta Pathol Microbiol Scand. 1968;72(4):586-600.

5. Joos RW, Hall WH. Neutralization of beta-lysin and cationic bactericidal agents by acidic phospholipid. J Bacteriol. 1968;95(1):9-13.

6. Varea J, Monterroso B, Sáiz JL, López-Zumel C, García JL, Laynez J, et al. Structural and thermodynamic characterization of $\mathrm{Pal}$, a phage natural chimeric lysin active against pneumococci. J Biol Chem. 2004;279(42):43697-707.

7. Traczewski MM, Ambler JE, Schuch R. Determination of MIC quality control parameters for exebacase, a novel lysin with antistaphylococcal activity. J Clin Microbiol. 2021;59(7):e0311720.

8. Indiani C, Sauve K, Raz A, Abdelhady W, Xiong YQ, Cassino C, et al. The antistaphylococcal lysin, CF-301, activates key host factors in human blood to potentiate methicillin-resistant. Antimicrob Agents Chemother. 2019;63(4):e022291-e22318.

9. Karau MJ, Schmidt-Malan SM, Yan Q, Greenwood-Quaintance KE, Mandrekar J, Lehoux D, et al. Exebacase in addition to daptomycin is more active than daptomycin or exebacase alone in methicillin-resistant Staphylococcus aureus osteomyelitis in rats. Antimicrob Agents Chemother. 2019;63(10):e01235-e1319.

10. Schuch R, Lee HM, Schneider BC, Sauve KL, Law C, Khan BK, et al. Combination therapy with lysin CF-301 and antibiotic is superior to antibiotic alone for treating methicillin-resistant Staphylococcus aureus-induced murine bacteremia. J Infect Dis. 2014;209(9):1469-78.

11. Fowler VG, Das AF, Lipka-Diamond J, Schuch R, Pomerantz R, Jáuregui-Peredo L, et al. Exebacase for patients with Staphylococcus aureus bloodstream infection and endocarditis. J Clin Invest. 2020;130(7):3750-60.

12. Barry M, Bari SA, Akhtar MY, Al Nahdi F, Erlandez R, Al Khushail A, et al. Clinical and microbiological characteristics of infective endocarditis at a cardiac center in Saudi Arabia. J Epidemiol Glob Health. 2021;11(4):435-43.

13. Grillo S, Cuervo G, Laporte-Amargós J, Tuells M, Grau I, Berbel $\mathrm{D}$, et al. Bloodstream infection and endocarditis caused by Staphylococcus aureus in patients with cancer: a multicenter cohort study. Infect Dis Ther. 2021. https://doi.org/10.1007/s40121-02100575-8 (Epub 2 Dec 2021).

14. Jordan AM, Tatum R, Ahmad D, Patel SV, Maynes EJ, Weber MP, et al. Infective endocarditis following heart transplantation: A systematic review. Transplant Rev (Orlando). 2021;36(1):100672.

15. Anstead GM, Quinones-Nazario G, Lewis JS. Treatment of infections caused by resistant Staphylococcus aureus. Methods Mol Biol. 2007;391:227-58.

16. Gao L, Tang Z, Li T, Wang J. Combination of kaempferol and azithromycin attenuates Staphylococcus aureus-induced osteomyelitis via anti-biofilm effects and by inhibiting the phosphorylation of ERK1/2 and SAPK. Pathog Dis. 2021;79(8):ftab048.

17. Lang S, Frömming A, Walter N, Freigang V, Neumann C, Loibl $\mathrm{M}$, et al. Is there a difference in clinical features, microbiological epidemiology and effective empiric antimicrobial therapy comparing healthcare-associated and community-acquired vertebral osteomyelitis? Antibiotics (Basel). 2021;10(11):1410.

18. Strange $S$, Whitehouse MR, Beswick AD, Board T, Burston A, Burston B, et al. One-stage or two-stage revision surgery for prosthetic hip joint infection - the INFORM trial: a study protocol for a randomised controlled trial. Trials. 2016;17:90. 
19. Minassian AM, Osmon DR, Berendt AR. Clinical guidelines in the management of prosthetic joint infection. J Antimicrob Chemother. 2014;69(Suppl 1):i29-35.

20. Lentino JR. Infections associated with prosthetic knee and prosthetic hip. Curr Infect Dis Rep. 2004;6(5):388-92.

21. Lentino JR. Prosthetic joint infections: bane of orthopedists, challenge for infectious disease specialists. Clin Infect Dis. 2003;36(9):1157-61.

22. Berbari EF, Hanssen AD, Duffy MC, Steckelberg JM, Ilstrup DM, Harmsen WS, et al. Risk factors for prosthetic joint infection: case-control study. Clin Infect Dis. 1998;27(5):1247-54.

23. Ferry T, Batailler C, Souche A, Cassino C, Chidiac C, Perpoint $\mathrm{T}$, et al. Arthroscopic "Debridement and Implant Retention" with local administration of exebacase (Lysin CF-301) followed by suppressive tedizolid as salvage therapy in elderly patients for relapsing multidrug-resistant. Front Med (Lausanne). 2021;8:550853.

24. Li WS, Chen YC, Kuo SF, Chen FJ, Lee CH. The impact of biofilm formation on the persistence of candidemia. Front Microbiol. 2018;9:1196.

25. Sherry L, Ramage G, Kean R, Borman A, Johnson EM, Richardson MD, et al. Biofilm-forming capability of highly virulent, multidrug-resistant Candida auris. Emerg Infect Dis. 2017;23(2):328-31.

26. Knafl D, Tobudic S, Cheng SC, Bellamy DR, Thalhammer F. Dalbavancin reduces biofilms of methicillin-resistant Staphylococcus aureus (MRSA) and methicillin-resistant Staphylococcus epidermidis (MRSE). Eur J Clin Microbiol Infect Dis. 2017;36(4):677-80.

27. Oh BJ, Shin JH, Kim MN, Sung H, Lee K, Joo MY, et al. Biofilm formation and genotyping of Candida haemulonii, Candida pseudohaemulonii, and a proposed new species (Candida auris) isolates from Korea. Med Mycol. 2011;49(1):98-102.

28. Elvers KT, Leeming K, Moore CP, Lappin-Scott HM. Bacterialfungal biofilms in flowing water photo-processing tanks. J Appl Microbiol. 1998;84(4):607-18.

29. Hamdan-Partida A, González-García S, de la Rosa GE, BustosMartínez J. Community-acquired methicillin-resistant Staphylococcus aureus can persist in the throat. Int J Med Microbiol. 2018;308(4):469-75.

30. Mohammadi M, Soroush S, Delfani S, Pakzad I, Abbaszadeh A, Bahmani M, et al. Distribution of class D carbapenemase and extended-spectrum $\beta$-lactamase genes among Acinetobacter baumannii isolated from burn wound and ventilator associated pneumonia infections. J Clin Diagn Res. 2017;11(7):DC19-23.

31. Larkin E, Hager C, Chandra J, Mukherjee PK, Retuerto M, Salem I, et al. The emerging pathogen Candida auris: growth phenotype, virulence factors, activity of antifungals, and effect of SCY-078, a novel glucan synthesis inhibitor, on growth morphology and biofilm formation. Antimicrob Agents Chemother. 2017;61(5):e02396-e2416.

32. Schuch R, Khan BK, Raz A, Rotolo JA, Wittekind M. Bacteriophage lysin CF-301, a potent antistaphylococcal biofilm agent. Antimicrob Agents Chemother. 2017;61(7):e02666-e2716.

33. Oh JT, Cassino C, Schuch R. Postantibiotic and sub-MIC effects of exebacase (Lysin CF-301) enhance antimicrobial activity against Staphylococcus aureus. Antimicrob Agents Chemother. 2019;63(6):e02616-e2618.

34. Watson A, Sauve K, Cassino C, Schuch R. Exebacase demonstrates in vitro synergy with a broad range of antibiotics against both methicillin-resistant and methicillin-susceptible Staphylococcus aureus. Antimicrob Agents Chemother. 2020;64(2):e01885-e1919.

35. Watson A, Oh JT, Sauve K, Bradford PA, Cassino C, Schuch R. Antimicrobial activity of exebacase (Lysin CF-301) against the most common causes of infective endocarditis. Antimicrob Agents Chemother. 2019;63(10):e01078-e1119.

36. Traczewski M, Oh J, Cassino C, Schuch R. In vitro activity of Exebacase (CF-301) against clinical Staphylococcus aureus surveillance isolates from the United States, Europe, and Latin America, 2015-2017. Diagn Microbiol Infect Dis. 2019;95(4):114879.

37. Swift SM, Sauve K, Cassino C, Schuch R. Exebacase is active. Antimicrob Agents Chemother. 2021;65(9):e0272320.

38. Shah SU, Xiong YQ, Abdelhady W, Iwaz J, Pak Y, Schuch R, et al. Effect of the lysin exebacase on cardiac vegetation progression in a rabbit model of methicillin-resistant Staphylococcus aureus endocarditis as determined by echocardiography. Antimicrob Agents Chemother. 2020;64(7):e00482-e520.

39. Oh JT, Ambler JE, Cassino C, Schuch R. Development of a broth microdilution method for exebacase susceptibility testing. Antimicrob Agents Chemother. 2021;65(7):e0258720.

40. Kebriaei R, Stamper KC, Lev KL, Morrisette T, AbdulMutakabbir JC, Schuch R, et al. Exebacase in addition to daptomycin against MRSA. Antimicrob Agents Chemother. 2021;65(11):e0012821.

41. Fischetti VA. Bacteriophage lytic enzymes: novel anti-infectives. Trends Microbiol. 2005;13(10):491-6.

42. Dhople VM, Nagaraj R. Conformation and activity of delta-lysin and its analogs. Peptides. 2005;26(2):217-25.

43. Goda K, Kenzaka T, Hoshijima M, Yachie A, Akita H. Toxic shock syndrome with a cytokine storm caused by Staphylococcus simulans: a case report. BMC Infect Dis. 2021;21(1):19.

44. Takayama Y, Yano H, Nojima Y, Nakano R, Okamoto R, Hirakata $\mathrm{Y}$, et al. Influence of prior pandemic A(H1N1)2009 virus infection on invasion of MDCK cells by community-associated methicillin-resistant Staphylococcus aureus. J Infect Chemother. 2014;20(1):71-3.

45. Kobayashi SD, Olsen RJ, LaCasse RA, Safronetz D, Ashraf M, Porter AR, et al. Seasonal H3N2 influenza A virus fails to enhance Staphylococcus aureus co-infection in a non-human primate respiratory tract infection model. Virulence. 2013;4(8):707-15.

46. Löffler B, Niemann S, Ehrhardt C, Horn D, Lanckohr C, Lina G, et al. Pathogenesis of Staphylococcus aureus necrotizing pneumonia: the role of PVL and an influenza coinfection. Expert Rev Anti Infect Ther. 2013;11(10):1041-51.

47. Robinson KM, Choi SM, McHugh KJ, Mandalapu S, Enelow RI, Kolls JK, et al. Influenza A exacerbates Staphylococcus aureus pneumonia by attenuating IL- $1 \beta$ production in mice. J Immunol. 2013;191(10):5153-9.

48. Robinson KM, McHugh KJ, Mandalapu S, Clay ME, Lee B, Scheller EV, et al. Influenza A virus exacerbates Staphylococcus aureus pneumonia in mice by attenuating antimicrobial peptide production. J Infect Dis. 2014;209(6):865-75.

49. Valour F, Richard JC, Chidiac C, Ferry T. Panton-Valentine leukocidin-positive staphylococcus aureus necrotising pneumonia complicating pandemic $\mathrm{A}(\mathrm{H} 1 \mathrm{~N} 1)$ influenza infection. BMJ Case Rep. 2013; p. bcr2013010227.

50. Fischetti V. Vincent Fischetti-following phages for life. Interview by Marilynn Larkin. Lancet Infect Dis. 2004;4(4):246-9. 